\title{
A Rare Case Report on Mediastinal Lipomatosis
}

Dr. Roopa Rajavarthini", Dr. A. Chithirai Selvam, Dr. R. Monika, Dr. M. Prabhakaran MDRD Department of Radiodiagnosis, Sree Balaji Medical College and Hospital CLC works road, Chromepet, Chennai, India

\author{
Article History \\ Received: 11.10 .2020 \\ Accepted: 24.10 .2020 \\ Published: 14.11.2020
}

Journal homepage:

https://www.easpublisher.com/easmb

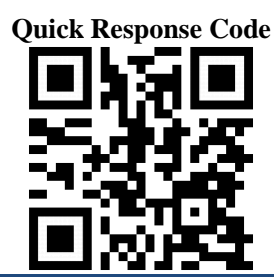

Abstract: Mediastinal lipomatosis is a rare condition characterised by a large amount of mature adipose tissue in the mediastinum. For 6 months, a case of dyspnea, breathlessness and cough, originally misdiagnosed as a case of right lower lobe pneumonia, was found to have mediastinal lipomatosis dependent on CT on further examination and validated with HPE association after excision. This case of ML occurred in the absence of steroid use, diabetes, the condition of Cushing. This case provides a major differential diagnosis of a patient with dyspnea. Potentially, this is one of the few instances of ML.

Keywords: Dyspnoea, mediastinal lipomatosis, dyslipidemia.

Copyright $($ C 2020 The Author(s): This is an open-access article distributed under the terms of the Creative Commons Attribution 4.0 International License (CC BY-NC 4.0) which permits unrestricted use, distribution, and reproduction in any medium for non-commercial use provided the original author and source are credited.

\section{INTRODUCTION}

A 70-year-old female presented to our institute with complaints of dyspnoea on exertion associated with cough for 6 months, there was no significant past medical history. She denied taking steroids in the past nor was she a known case of Cushing's disease.

\section{EXPERIMENTS AND METHODS}

On physical examination, we observed an aged woman, who was found to be obese, with increased waist-hip ratio. Her Chest auscultation and local examination of the breast bilateral was normal. Did not reveal any significant abnormality. Her lipid profile was suboptimal, revealed mildly elevated cholesterol $(222 \mathrm{mg} / \mathrm{dL})$ and triglycerides $(197 \mathrm{mg} / \mathrm{dL})$ and other blood investigations were within normal limits. Sputum AFB culture was found to be negative.

Routine $\mathrm{x}$ ray showed hazy opacity in the lower zone of right lung field, with normal cardiac borders and bilateral hemidiaphragms were normal.

She was diagnosed with right lower lobe pneumonia and was treated for the same, after one week of treatment a repeat X-ray was taken, which did not show any improvement from the previous one. A contrast enhanced computerised tomogram was ordered which showed a well-defined, non-enhancing encapsulated fat attenuated mass with few strands of soft tissue in the anterior mediastinum more on the right side, crossing the midline causing mild shift of the heart towards the left side - features suggestive of mediastinal lipomatosis

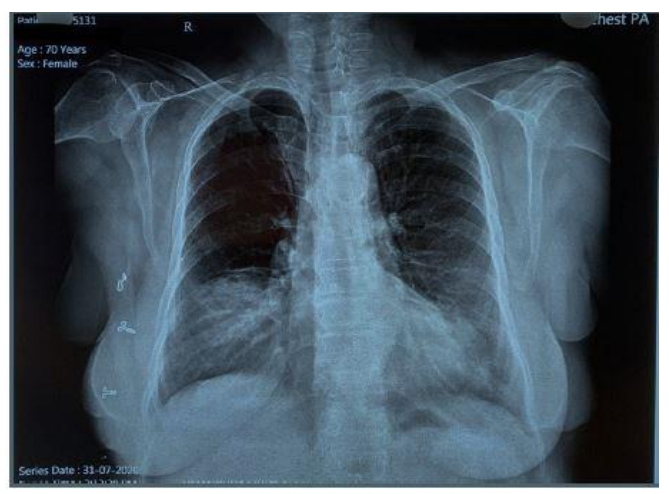

Fig-1a: $x$ ray PA view of chest showing a hazy opacity involving the lower lobe of right lung 

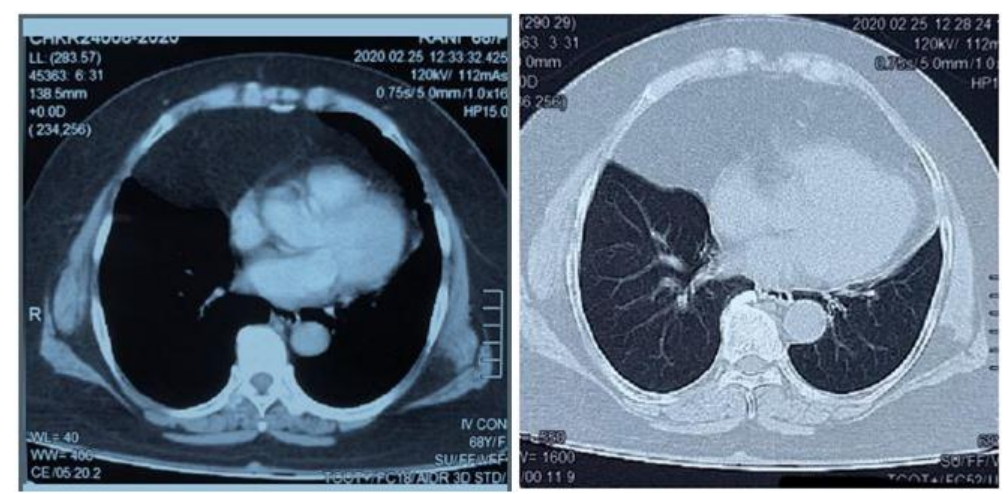

Fig-2: A and B - CECTof chest mediastinal window ( A) and lung window ( B) axial section, showing a well-defined, non-enhancing encapsulated fatty mass in the anterior mediastinum, predominantly on the right side, extending across the midline

Rest of the lung fields was unremarkable. Following this, the rest of the lung areas were unremarkable. Subsequent to this, CT guided lung biopsy performed, which yielded few clinical samples. The sample was submitted for histopathological analysis. Thus, the sample showed it to be (1 ipoma) lipomatosis. Finally, the diagnosis of mediastinal lipomatosis with dyslipdemia was then made.

The patient was admitted for surgery in which lipomatosis visualised, was excised from the anterior mediastinum. The specimen was sent for histopathological analysis. Histological study demonstrated lobules of mature adipose tissue with a fibrous capsule. There was no evidence of malignancy. Post-operative period was uneventful, and the patient was discharged in 10 days.

\section{DisCUSSION}

There are various fat containing lesions of the chest including parenchymal and endobronchial lesions such as hamartoma, lipoid pneumonia and lipoma. Mediastinal fat containing lesions include germ cell neoplasm. Lipomatosis refers to excessive deposition of encapsulated fat in the mediastinum. As alluded above, the patient was diagnosed with mediastinal lipomatosis but had no history of thoracic trauma, obesity, Cushing's syndrome or chronic steroid intake, which are medical conditions known to be associated with mediastinal lipoma. Mediastinal lipomas resemble other soft-tissue masses on chest radiography and are commonly categorised by location as usually at the cardio diaphragmatic angle, cervicomediastinal or transmural. For differential diagnosis between the cardiac and extra-cardiac causes of mediastinal enlargement, CT is obligatory. On CT, lipomas have homogenous UN encapsulated fat attenuating region of approximately $-100 \mathrm{HU}$, involving the mediastinum, which will sharply outline vessels and lymph nodes. CT with intravenous contrast has conventionally been the imaging modality of choice in the evaluation and categorisation of an anterior mediastinal mass. The use of CT imaging is extremely beneficial in the evaluation of fatty lesions in the thorax. When such lesions are detected, identification of their location and imaging characteristics significantly reduces the time required for differential diagnosis.

\section{REFERENCES}

1. Nguyen, K.Q., Hoeffel, C., Le, L.H., Phan, H.T. (1998). Mediastinal lipomatosis. South Med J; 91:1169-72.

2. Jamali, M., Zouhair, K., El Ouazzani, T., \& Lakhdar, H. (2006, March). Precocious mediastinal lipomatosis: a rare complication of systemic corticosteroid therapy. In Annales de dermatologie et de venereologie (Vol. 133, No. 3, p. 257).

3. Kashikar, S., Gulkari, A. J., \& Singhania, P. K. (2012). CT to the rescue in benign, symmetrical mediastinal lipomatosis. Thorax, 67(8), 758-758.

4. Mohapatra, P. R., \& Janmeja, A. K. (2010). Asymptomatic mediastinal lipomatosis. New England Journal of Medicine, 363(13), 1265-1265.

5. Cutilli, T., Schietroma, M., Marcelli, V., Ascani, G., \& Corbacelli, A. (1999). Giant cervicomediastinal lipoma. A clinical case. Minerva Stomatol., 48.

6. Lee, W. J., \& Fattal, G. (1976). Mediastinal lipomatosis in simple obesity. Chest, 70(2), 308309.

7. Sorhage, F., Stover, D. E., \& Mortazavi, A. (1996). Unusual etiology of cough in a woman with asthma. Chest, 110(3), 852-854.

8. Carter, B. W., Marom, E. M., \& Detterbeck, F. C. (2014). Approaching the patient with an anterior mediastinal mass: a guide for clinicians. Journal of Thoracic Oncology, 9(9), S102-S109. 